Revista Brasileira de Odontologia Legal - RBOL

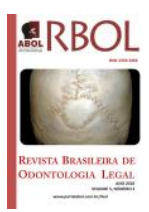

Odontologia Legal

\title{
MAPEAMENTO DAS REVISTAS CIENTÍFICAS NACIONAIS E INTERNACIONAIS E SUAS PRODUÇÕES NA ÁREA DA ODONTOLOGIA LEGAL.
}

\section{Mapping of nationals and internationals scientific journals and productions in the area of Forensic Dentistry.}

\author{
Ana Luísa Rezende MACHADO ${ }^{1}$, Tamara Soledad Frontanilla RECALDE ${ }^{1}$, \\ Silmara Pereira de SOUSA ${ }^{2}$, Ricardo Henrique Alves da SILVA ${ }^{3}$.
}

1. Aluna de Mestrado em Patologia e Medicina Legal. Departamento de Patologia e Medicina Legal. Faculdade de Medicina de Ribeirão Preto. Universidade de São Paulo. São Paulo. Brasil.

2. Aluna do Curso de Especialização em Odontologia Legal. Departamento de Estomatologia, Saúde Coletiva e Odontologia Legal. Área de Odontologia Legal. Faculdade de Odontologia de Ribeirão Preto. Universidade de São Paulo. São Paulo. Brasil.

3. Professor Doutor da Faculdade de Odontologia de Ribeirão Preto. Departamento de Estomatologia, Saúde Coletiva e Odontologia Legal. Área de Odontologia Legal. Faculdade de Odontologia de Ribeirão Preto. Universidade de São Paulo. São Paulo. Brasil.

Informação sobre o manuscrito

Recebido em: 31 Julho 2018

Aceito em: 13 Agosto 2018

\author{
Autor para contato: \\ Prof. Dr. Ricardo Henrique Alves da Silva \\ Av. do Café, $s / n-$ Campus da USP \\ Ribeirão Preto, São Paulo, Brasil. \\ CEP: $14040-904$ \\ E-mail: ricardohenrique@usp.br.
}

\section{RESUMO}

Publicações periódicas de artigos científico-acadêmicos buscam a transferência de informação e construção do conhecimento de alta qualidade. A Odontologia Legal, apesar do amplo campo de atuação profissional, envolve vários temas referentes à legislação, bem como especificidades locais, questões essas que, muitas vezes, dificultam a publicação em revistas científicas com alto fator de impacto. Visto isso, o presente estudo objetivou mapear as revistas científicas nacionais e internacionais que apresentem publicações na área da Odontologia Legal, bem como expor as dificuldades existentes na publicação de trabalhos relacionados à área em revistas de maio relevância. Foi acessada a Plataforma Sucupira e selecionada a Odontologia como área de avaliação e, assim, obtido um arquivo de classificações. Todas as revistas que apresentaram temas relacionados à Odontologia Legal foram registradas em duas tabelas com a quantidade de artigos publicados relacionados à área em cada uma delas. Com bases nos critérios de busca, foram encontradas 191 revistas, sendo 75 nacionais e 116 internacionais. As publicações nacionais mostraram escassa produção em revistas com fator de alto impacto, enquanto que as internacionais apresentaram uma maior quantidade, apesar de ser minoria dentro da produção total. Com esse trabalho, foi possível comparar a classificação Qualis entre revistas nacionais e internacionais, destacando a distância entre tais classificações e expondo a dificuldade de publicação em revistas de alta competitividade e internacionalização.

\section{PALAVRAS-CHAVE}

Fator de impacto; Legislação; Odontologia legal; Pesquisa; Publicações periódicas. 


\section{INTRODUÇÃO}

Publicações periódicas de artigos científico-acadêmicos, nas mais diversas revistas científicas, buscam a transferência de informação e construção do conhecimento de alta qualidade, validade e credibilidade, divulgando resultados de pesquisas, resenhas, resumos de dissertações e outras modalidades, de forma fácil, econômica e rápida. Concedem, assim, conhecimentos àqueles que desejam inteirar-se sobre os avanços da ciência e conferindo a oportunidade aos pesquisadores de relatar os resultados de estudos e pesquisas nas mais diversas áreas ${ }^{1,2}$.

Atualmente, as revistas científicas podem ser produzidas de três formas, por editoras comerciais, as quais determinam, através de renomados revisores, a publicação, ou não, de artigos previamente submetidos, os quais passam a pertencer às editoras e, para se ter acesso aos mesmos, a compra da assinatura é exigida. A segunda variedade de revista é aquela produzida por Universidades, Associações, Sociedades de classes profissionais ou outras entidades sem fins lucrativos, sendo solicitada uma anuidade ou mensalidade aos seus sócios, com exceção das Universidades que editam alguns periódicos distribuídos de forma gratuita. A terceira forma de publicação de revistas científicas se dá por meio de publicações eletrônicas e pela Internet, ou seja, revistas online de acesso gratuito, nas quais os periódicos não possuem direitos autorais sobre os artigos ${ }^{1}$.

Os artigos veiculados unicamente por meio eletrônico que, muitas vezes, não obedecem a um sistema rigoroso de revisão que Ihes confira a devida qualidade e a grande pressão empregada pelas instituições de ensino e pelos órgãos de fomento no quesito publicação, leva a grande proliferação de revistas científicas, resultando em problemas como o de credibilidade e qualidade, além da questionada disponibilidade duradoura, uma vez que a obsolescência tecnológica está envolvida neste quesito, embora tenha se tornado uma forma rápida, de fácil acesso e de baixo custo $^{1-3}$.

Dessa forma, nota-se a necessidade de se estabelecer um sistema de classificação dos inúmeros periódicos nacionais e internacionais de todas as áreas do conhecimento. A Coordenação de Aperfeiçoamento de Pessoa de Nível Superior (CAPES), vinculada ao Ministério da Educação (Brasil), oferece em sua Plataforma Sucupira uma lista de diversos periódicos científicos, avaliados de quatro em quatro anos, denominada Qualis, a qual é realizada pelos comitês de consultores de cada área de avaliação, seguindo critérios previamente definidos, como um elevado fator de impacto em bases de dados reconhecidas, periodicidade, competitividade e internacionalidade ${ }^{4,5}$.

$O$ reconhecimento do trabalho e 0 alcance do impacto esperado dos resultados do estudo são primordiais para o sucesso da pesquisa científica, logo, tornase necessário que os pesquisadores saibam quais são as revistas mais apropriadas para sua área, ou seja, aquelas que publicam artigos significativos e interessantes, seguindo um rigor nas avaliações com seus revisores qualificados e elevando o fator de impacto da publicação ${ }^{5,6}$. 
A Odontologia Legal, em particular, é a especialidade odontológica que trabalha diretamente com a Justiça, podendo atuar nas esferas cível, criminal e trabalhista, compreendendo as perícias no vivo, morto, nas ossadas, em fragmentos, em trabalhos odontológicos isolados e até mesmo em peças dentais isoladas. Desse modo, o Odontolegista atua em processos de identificação humana em casos de acidentes em massa, corpos carbonizados ou em avançado estado de decomposição, estimativa de idade para averiguação da maioridade, em cenas de crimes ou maus tratos a crianças e idosos, em processos de erros profissionais, auditorias e até mesmo em perícias administrativas nos conselhos de classe ${ }^{7-10}$.

Todavia, apesar desta vasta gama de atuação dos cirurgiões-dentistas especialistas em Odontologia Legal, é indispensável destacar a importância do conhecimento sobre legislações e normativas mesmo no ambiente clínico odontológico, pela necessidade de elaboração de documentos como prontuários e encaminhamentos ${ }^{8,9}$.

Visto isso, torna-se necessário o conhecimento de revistas ligadas à Odontologia Legal para maior compreensão e entendimento dos cirurgiões-dentistas que atuam no ambiente clínico, além daqueles ligados à especialidade, como em casos de professores, peritos ou auditores, e consequentemente, concede a oportunidade aos pesquisadores de relatar os resultados de seus estudos e pesquisas. $O$ presente estudo visa mapear as revistas científicas nacionais e internacionais com publicações na área da Odontologia Legal, bem como expor as dificuldades existentes na publicação de trabalhos relacionados à área em revistas de alto fator de impacto.

\section{MATERIAL E MÉTODOS}

Para compor a amostra com todas as revistas nacionais e internacionais, foi acessada a Plataforma Sucupira (https://sucupira.capes.gov.br/sucupira/publi c/consultas/coleta/veiculoPublicacaoQualis/li staConsultaGeralPeriodicos.jsf) no dia 12 de dezembro de 2017, na qual há a classificação de acordo com os indicativos da qualidade do sistema Qualis-Periódicos: A1, A2, B1, B2, B3, B4, B5 e C, sendo a classificação $A 1$ atribuída como o nível mais elevado da produção intelectual e C, o mais baixo. Foi selecionada a Odontologia como área de avaliação e assim, obtido um arquivo de classificações ${ }^{4}$.

Por meio do arquivo referente às classificações, o site de cada uma dessas revistas foi acessado durante o período de 12 de dezembro de 2017 a 2 de fevereiro de 2018 por três pesquisadores, onde um ficou responsável pelas revistas nacionais e os outros dois pelas internacionais, por estas possuírem um volume maior de publicações. Foram utilizadas as palavras-chaves "forensic dentistry", "forensic odontology" e "law", visto que as mesmas abrangem diversos assuntos pertencentes à especialidade, como antropologia forense e legislação, aumentando o número de artigos encontrados. Dessa forma, foram selecionados os periódicos que apresentaram temas relacionados à Odontologia Legal, tanto em seus objetivos e escopo, quanto em suas publicações, independentemente de seu país de origem. 
As revistas nacionais foram registradas em uma planilha e as revistas internacionais em uma segunda planilha, ambas juntamente com outros dados, como país de origem, segundo o catálago da US National Library of Medicine (NLM), número do volume atual, ISSN, qualis e número de artigos publicados na área em questão ${ }^{11}$.
Foram encontradas 191 revistas com publicações relacionadas à Odontologia Legal, das quais 75 constituem revistas nacionais e 116 internacionais, representadas nas Tabelas 1 e 2. As revistas foram ordenadas de acordo com a classificação Qualis (Tabela 1 e 2).

\section{RESULTADOS}

Tabela 1. Revistas nacionais ordenadas de acordo com a classificação Qualis (2013 - 2016).

\begin{tabular}{|c|c|c|c|}
\hline Nome Da Revista & Volumes & Qualis & $\begin{array}{c}\text { Artigos } \\
\text { Publicados }\end{array}$ \\
\hline Brazilian Dental Journal & 29 & A2 & 4 \\
\hline Brazilian Oral Research & 32 & $\mathrm{~A} 2$ & 7 \\
\hline Journal Of Applied Oral Science & 26 & $\mathrm{~A} 2$ & 6 \\
\hline Genetics and Molecular Biology & 40 & B1 & 7 \\
\hline Anais da Academia Brasileira de Ciências & 89 & B2 & 3 \\
\hline Brazilian Journal of Medical and Biological Research & 51 & B2 & 0 \\
\hline Genetics and Molecular Research & 16 & B2 & 3 \\
\hline Acta Scientiarum - Health Sciences & 39 & B3 & 3 \\
\hline Bioscience Journal & 34 & B3 & 6 \\
\hline Brazilian Journal of Oral Sciences & 15 & B3 & 6 \\
\hline Journal of Health Science (UNOPAR) & 19 & B3 & 1 \\
\hline Medicina (Ribeirão Preto) & 50 & B3 & 2 \\
\hline Revista da ABENO & 17 & B3 & 7 \\
\hline Revista de Odontologia da UNESP & 46 & B3 & 6 \\
\hline Revista Odonto Ciência & 32 & B3 & 1 \\
\hline RGO - Revista Gaúcha de Odontologia & 65 & B3 & 4 \\
\hline Saúde em Debate & 41 & B3 & 4 \\
\hline Saúde e Sociedade & 26 & B3 & 4 \\
\hline Archives of Oral Research & 9 & B4 & 1 \\
\hline Arquivo Brasileiro de Odontologia & 12 & B4 & 3 \\
\hline Arquivos Brasileiros de Ciências da Saúde & 37 & B4 & 0 \\
\hline Arquivos de Ciências da Saúde (FAMERP) & 24 & B4 & 0 \\
\hline Arquivos em Odontologia (UFMG) & 53 & B4 & 6 \\
\hline Brazilian Dental Science & 20 & B4 & 3 \\
\hline $\begin{array}{l}\text { Brazilian Journal of Forensic Sciences, Medical Law and } \\
\qquad \text { Bioethics }\end{array}$ & 7 & B4 & 8 \\
\hline Clinical and Laboratorial Research in Dentistry & 21 & B4 & 2 \\
\hline Hu Revista & 42 & B4 & 0 \\
\hline Jornal Interdisciplinar de Biociências & 2 & B4 & 0 \\
\hline Journal of Clinical Dentistry and Research & 14 & B4 & 1 \\
\hline
\end{tabular}




\begin{tabular}{|c|c|c|c|}
\hline Nome Da Revista & Volumes & Qualis & $\begin{array}{c}\text { Artigos } \\
\text { Publicados }\end{array}$ \\
\hline Odonto (UMESP) & 24 & B4 & 16 \\
\hline Odontologia Clínico-Científica & 15 & B4 & 7 \\
\hline Revista Brasileira de Ciências da Saúde & 21 & B4 & 8 \\
\hline Revista Brasileira de Criminalística & 6 & B4 & 32 \\
\hline Revista Brasileira de Odontologia & 74 & B4 & 1 \\
\hline Revista Brasileira de Odontologia Legal & 4 & B4 & 88 \\
\hline Revista Brasileira de Pesquisa em Saúde & 19 & B4 & 0 \\
\hline Revista Ciência em Extensão & 13 & B4 & 1 \\
\hline Revista Ciência Plural & 3 & B4 & 0 \\
\hline Revista Ciências Médicas e Biológicas & 16 & B4 & 7 \\
\hline Revista Conexão UEPG & 14 & B4 & 1 \\
\hline Revista Cultura e Extensão USP & 17 & B4 & 0 \\
\hline Revista da APCD & 70 & B4 & 6 \\
\hline Revista da Faculdade de Ciências Médicas de Sorocaba & 19 & B4 & 0 \\
\hline $\begin{array}{l}\text { Revista da Faculdade de Odontologia da Universidade de } \\
\qquad \text { Passo Fundo }\end{array}$ & 22 & B4 & 16 \\
\hline $\begin{array}{l}\text { Revista da Faculdade de Odontologia da Universidade Federal } \\
\qquad \text { da Bahia }\end{array}$ & 46 & B4 & 0 \\
\hline Revista da Faculdade de Odontologia de Lins & 27 & B4 & 2 \\
\hline Revista da Faculdade de Odontologia de Porto Alegre & 58 & B4 & 2 \\
\hline Revista da Universidade Vale do Rio Verde & 16 & B4 & 3 \\
\hline Revista de Ciências da Saúde (São Luís) & 18 & B4 & 0 \\
\hline Revista de Ciências Médicas (PUCCAMP) & 26 & B4 & 0 \\
\hline Revista de Odontologia (USP) & 13 & B4 & 0 \\
\hline Revista de Odontologia da UNICID & 29 & B4 & 0 \\
\hline Revista de Pesquisa em Saúde & 18 & B4 & 3 \\
\hline Revista do Instituto de Ciências da Saúde (UNIP) & 35 & B4 & 0 \\
\hline Revista Eletrônica Acervo Saúde & 10 & B4 & 0 \\
\hline Revista Paraense de Odontologia & 2 & B4 & 1 \\
\hline Revista Saúde \& Ciência & 6 & B4 & 0 \\
\hline Revista Saúde (UNG) & 11 & B4 & 0 \\
\hline Revista Uniabeu & 10 & B4 & 0 \\
\hline Revista Sul-Brasileira de Odontologia & 14 & B4 & 8 \\
\hline Saúde (Santa Maria) & 43 & B4 & 1 \\
\hline Saúde e Pesquisa & 10 & B4 & 0 \\
\hline Saúde, Ética \& Justiça & 22 & B4 & 20 \\
\hline Journal of Research in Dentistry & 5 & B5 & 3 \\
\hline Oral Sciences & 6 & B5 & 0 \\
\hline Revista Bahiana de Odontologia & 8 & B5 & 4 \\
\hline Revista Ciência e Saúde & 2 & B5 & 0 \\
\hline Revista Coopex & 8 & B5 & 0 \\
\hline Revista Cpaqv & 10 & B5 & 0 \\
\hline
\end{tabular}




\begin{tabular}{cccc}
\hline Nome Da Revista & Volumes & Qualis & $\begin{array}{c}\text { Artigos } \\
\text { Publicados }\end{array}$ \\
\hline Revista de Iniciação Científica em Odontologia & 15 & B5 & 0 \\
Revista do CROMG & 17 & B5 & 0 \\
Revista Odontológica de Araçatuba & 38 & B5 & 0 \\
Revista Science in Health & 5 & B5 & 0 \\
Revista Virtual da Academia Brasileira de Odontologia & 8 & B5 & 1 \\
Scientific Investigation in Dentistry & 22 & B5 & 0 \\
TOTAL & 1803 & & 329 \\
\hline
\end{tabular}

Tabela 2. Revistas internacionais ordenadas de acordo com a classificação Qualis (2013-2016).

\begin{tabular}{|c|c|c|c|c|}
\hline Nome Da Revista & Pais & Volumes & Qualis & $\begin{array}{c}\text { Artigos } \\
\text { Publicados }\end{array}$ \\
\hline American Journal Of Human Genetics & EUA & 101 & A1 & 0 \\
\hline Bmc Genomics & Inglaterra & 19 & A1 & 0 \\
\hline Clinical Oral Investigations & Alemanha & 22 & A1 & 4 \\
\hline Community Dentistry And Oral Epidemiology & Dinamarca & 45 & $\mathrm{~A} 1$ & 8 \\
\hline Forensic Science International. Genetics (Print) & Holanda & 32 & A1 & 2 \\
\hline Human Genetics & Alemanha & 137 & A1 & 1 \\
\hline Journal Of Dental Research & EUA & 97 & $\mathrm{~A} 1$ & 7 \\
\hline Journal Of Dentistry & Inglaterra & 68 & A1 & 10 \\
\hline Plos Genetics & EUA & 14 & $\mathrm{~A} 1$ & 0 \\
\hline American Journal Of Dentistry & EUA & 30 & $\mathrm{~A} 2$ & - \\
\hline Archives Of Oral Biology & Inglaterra & 88 & $\mathrm{~A} 2$ & 14 \\
\hline Forensic Science International & Irlanda & 284 & A2 & 132 \\
\hline International Journal Of Legal Medicine (Print) & Alemanha & 132 & A2 & 48 \\
\hline International Journal Of Oral Science & Índia & 9 & A2 & 0 \\
\hline The Journal Of The American Dental Association (1939) & Inglaterra & 149 & A2 & 41 \\
\hline Acta Odontologica Scandinavica & Inglaterra & 76 & B1 & 26 \\
\hline Australian Dental Journal (Print) & Austrália & 62 & B1 & 17 \\
\hline Bioscience Trends & Japão & 11 & B1 & 0 \\
\hline Bmc Health Services Research (Online) & Inglaterra & 18 & B1 & 0 \\
\hline Bmc Oral Health & Inglaterra & 18 & B1 & 7 \\
\hline British Journal Of Oral \& Maxillofacial Surgery & Escócia & 56 & B1 & 4 \\
\hline European Journal Of Oral Sciences & Inglaterra & 125 & B1 & 7 \\
\hline International Journal Of Dentistry & Egito & 10 & B1 & 4 \\
\hline International Dental Journal & Inglaterra & 68 & B1 & 5 \\
\hline Legal Medicine & Japão & 30 & B1 & 5 \\
\hline Medicine (Baltimore) & EUA & 97 & B1 & 2 \\
\hline Odontology & Japão & 106 & B1 & 3 \\
\hline Science \& Justice & Inglaterra & 58 & B1 & 4 \\
\hline Academy Of General Dentistry & EUA & 65 & B2 & 0 \\
\hline Advances In Dental Research & EUA & 29 & B2 & 1 \\
\hline
\end{tabular}




\begin{tabular}{|c|c|c|c|c|}
\hline Nome Da Revista & Pais & Volumes & Qualis & $\begin{array}{c}\text { Artigos } \\
\text { Publicados }\end{array}$ \\
\hline Anatomical Science International & Japão & 93 & B2 & 1 \\
\hline Annals Of Anatomy & Alemanha & 216 & B2 & 2 \\
\hline Australian Journal Of Forensic Science (Print) & Austrália & 50 & B2 & 24 \\
\hline British Dental Journal & Inglaterra & 224 & B2 & 49 \\
\hline Bulletin Of Tokyo Dental College & Japão & 58 & B2 & 1 \\
\hline Community Dental Health & Inglaterra & 34 & B2 & 1 \\
\hline European Journal Of Dental Education & Inglaterra & 21 & B2 & 7 \\
\hline European Journal Of Dentistry & Índia & 11 & B2 & 6 \\
\hline Genomics Data & EUA & 14 & B2 & 0 \\
\hline Imaging Science In Dentistry & $\begin{array}{l}\text { Coréia do } \\
\text { Sul }\end{array}$ & 47 & B2 & 3 \\
\hline Indian Journal Of Dental Research & Índia & 28 & B2 & 21 \\
\hline Journal Of Clinical And Experimental Dentistry & Espanha & 10 & B2 & 0 \\
\hline Journal Of Dental Sciences & Holanda & 12 & B2 & 2 \\
\hline Journal Of Forensic And Legal Medicine & Inglaterra & 53 & B2 & 22 \\
\hline Journal Of Forensic Odonto-Stomatology & Austrália & 32 & B2 & 348 \\
\hline Journal Of Forensic Radiology And Imaging & Holanda & 11 & B2 & 6 \\
\hline Journal Of Forensic Sciences & EUA & 63 & B2 & 173 \\
\hline Journal Of Investigative And Clinical Dentistry (Online) & Austrália & 8 & B2 & 4 \\
\hline Journal Of Oral Science & Japão & 59 & B2 & 0 \\
\hline Ohdm - Oral Health And Dental Management & EUA & 16 & B2 & 5 \\
\hline $\begin{array}{l}\text { The American Journal Of Forensic Medicine And } \\
\text { Pathology }\end{array}$ & EUA & 38 & B2 & 23 \\
\hline The Open Dentistry Journal & Holanda & 12 & B2 & 3 \\
\hline Acta Bioethica (Impresa) & Chile & 23 & B3 & 12 \\
\hline Acta Odontologica Latinoamericana & Argentina & 30 & B3 & 0 \\
\hline Acta Odontologica Venezolana & Venezuela & 55 & B3 & 13 \\
\hline Acta Stomatologica Croatica & Croácia & 51 & B3 & 2 \\
\hline Ces Odontologia & Colômbia & 30 & B3 & 3 \\
\hline Clinical And Experimental Dental Research & EUA & 3 & B3 & 3 \\
\hline Dental Research Journal & Irã & 15 & B3 & 6 \\
\hline Dentistry Today & EUA & 36 & B3 & 11 \\
\hline European Journal Of General Dentistry & Índia & 7 & B3 & 5 \\
\hline Indian Journal Of Dentistry & Índia & 7 & B3 & 3 \\
\hline $\begin{array}{l}\text { Journal Of Dentistry Of Tehran University Of Medical } \\
\qquad \text { Sciences }\end{array}$ & Irã & 15 & B3 & 1 \\
\hline Journal Of Forensic Dental Sciences & Índia & 9 & B3 & 198 \\
\hline Journal Of International Dental And Medical Research & Turquia & 10 & B3 & 2 \\
\hline Journal Of International Oral Health & Índia & 9 & B3 & 4 \\
\hline Journal Of Oral And Maxillofacial Research & Lituânia & 8 & B3 & 0 \\
\hline Journal Of Orofacial Sciences & Índia & 9 & B3 & 4 \\
\hline New York State Dental Journal & EUA & 83 & B3 & 0 \\
\hline
\end{tabular}




\begin{tabular}{|c|c|c|c|c|}
\hline Nome Da Revista & Pais & Volumes & Qualis & $\begin{array}{c}\text { Artigos } \\
\text { Publicados }\end{array}$ \\
\hline Persona Y Bioetica & Colômbia & 21 & B3 & 21 \\
\hline Revista Ciencias De La Salud & Colômbia & 15 & B3 & 0 \\
\hline Revista Cubana De Estomatologia & Cuba & 54 & B3 & 2 \\
\hline $\begin{array}{l}\text { Revista De La Facultad De Odontologia Universidad De } \\
\qquad \text { Antioquia }\end{array}$ & Colômbia & 29 & B3 & 4 \\
\hline World Journal Of Dentistry & Índia & 8 & B3 & 3 \\
\hline $\begin{array}{c}\text { Actas Odontológicas (Facultad De Odontología De La } \\
\text { Universidad Católica Del Uruguay) }\end{array}$ & Uruguai & 14 & B4 & 0 \\
\hline Eidon: Revista Espanhola De Bioética & Espanha & 48 & B4 & 0 \\
\hline Indian Journal Of Contemporary Dentistry & Índia & 5 & B4 & 4 \\
\hline $\begin{array}{l}\text { International Journal Of Dentistry And Oral Science } \\
\qquad \text { (ljdos) }\end{array}$ & EUA & 5 & B4 & 2 \\
\hline International Journal Of Health Sciences & EUA & 12 & B4 & 0 \\
\hline Journal Of Dental Research And Review & Índia & 4 & B4 & 5 \\
\hline Journal Of Forensic Investigation & EUA & 5 & B4 & 3 \\
\hline $\begin{array}{l}\text { Journal Of Interdisciplinary Medicine And Dental } \\
\text { Science }\end{array}$ & EUA & 5 & B4 & 0 \\
\hline Journal Of Science & Índia & 8 & B4 & 0 \\
\hline Odontología Sanmarquina & Peru & 12 & B4 & 5 \\
\hline Odovtos - International Journal Of Dental Sciences & Costa Rica & 20 & B4 & 2 \\
\hline Revista De La Asociación Odontológica Argentina & Argentina & 105 & B4 & 1 \\
\hline Revista De La Federacion Odontologica Colombiana & Colômbia & 75 & B4 & 1 \\
\hline Revista Dental De Chile & Chile & 107 & B4 & 0 \\
\hline Revista Ustasalud & Colômbia & 13 & B4 & 1 \\
\hline $\begin{array}{l}\text { American International Journal Of Ontemporary } \\
\text { Research }\end{array}$ & EUA & 8 & B5 & 0 \\
\hline Dental, Oral And Craniofacial Research & Inglaterra & 4 & B5 & 0 \\
\hline Dentistry 3000 & EUA & 5 & B5 & 2 \\
\hline $\begin{array}{l}\text { European International Journal Of Science And } \\
\qquad \text { Technology }\end{array}$ & Inglaterra & 7 & B5 & 0 \\
\hline European Journal Of Forensic Sciences (Print) & Inglaterra & 4 & B5 & 10 \\
\hline II Dentista Moderno & Itália & 36 & B5 & 17 \\
\hline International Journal Of Dental Science And Research & EUA & 6 & B5 & 2 \\
\hline International Journal Of Dentistry And Oral Health & EUA & 3 & B5 & 1 \\
\hline International Journal Of Oral And Dental Health & EUA & 4 & B5 & 0 \\
\hline International Journal Of Recent Scientific Research & Índia & 9 & B5 & 0 \\
\hline Italian Journal Of Dental Medicine & Itália & 2 & B5 & 0 \\
\hline Jacobs Journal Of Forensic Science & EUA & 1 & B5 & 1 \\
\hline Journal Of Dental And Oral Health & Canadá & 3 & B5 & 0 \\
\hline Journal Of Dental And Orofacial Research & Índia & 13 & B5 & 1 \\
\hline Journal Of Dentistry Indonesia & Indonésia & 24 & B5 & 2 \\
\hline Journal Of Forensic Science \& Criminology & EUA & 5 & B5 & 10 \\
\hline
\end{tabular}




\begin{tabular}{ccccc}
\hline Nome Da Revista & Pais & Volumes & Qualis & $\begin{array}{c}\text { Artigos } \\
\text { Publicados }\end{array}$ \\
\hline Jsm Dentistry & & & & B \\
Research \& Reviews: Journal Of Dental Sciences & Índia & 5 & B5 & 2 \\
Revista Portuguesa Do Dano Corporal & Portugal & 26 & B5 & 30 \\
Scientific Journal Of Dentistry & Índia & 2 & B5 & 0 \\
Annals Of Forensic Research And Analysis & EUA & 4 & C & 5 \\
Dentistry Journal & Suíça & 6 & C & 1 \\
Forensic Medicine And Anatomy Research & EUA & 6 & C & 4 \\
Jacobs Journal Of Dentistry And Research & EUA & 4 & C & 0 \\
Journal Of Forensic Research & EUA & 8 & C & 3 \\
Oa Dentistry & Inglaterra & 2 & C & 0 \\
Oral Biology And Dentistry & Índia & 5 & C & 0 \\
TOTAL & & 4338 & & 1408 \\
\hline
\end{tabular}

Apesar da grande quantidade de revistas científicas encontradas que aceitam publicações relacionadas à Odontologia legal, $33,16 \%$ não possuem trabalhos relacionados publicados. As publicações nacionais referentes à área supracitada, mostraram escassa produção em revistas com alto fator de impacto. Já o volume das publicações em revistas internacionais de alto impacto é maior, porém comparando o número total de artigos publicados em cada revista, é possível observar que representam uma minoria dentro da produção total. Como ocorre na revista "Forensic Science International", com um total de 9.710 artigos publicados na base de dados da PubMed, mas com apenas 1,35\% dessas publicações voltadas à área de Odontologia Legal. A mesma situação acontece com o "Journal of Forensic Science" com apenas $0,11 \%$ da sua produção total voltada à área supracitada ${ }^{12}$.

Como pode-se observar na Tabela 1 , as revistas brasileiras com mais enfoque na área de Odontologia Legal são: "Brazilian Journal of Forensic Sciences", "Medical Law and Bioethics", Revista Brasileira de Odontologia Legal e Revista Brasileira de Criminalística, todas classificadas como Qualis B4. A revista brasileira com Qualis A2 que mais se destaca é a "Brazilian Oral Research" com temas que abrangem a Odontologia de forma geral, e as revistas com Qualis B1 e B2 mais evidenciadas são: "Genetics and Molecular Biology" e "Genetics and Molecular Research", ambas com assuntos em Ciências Biológicas.

Já as revistas internacionais que apresentam maior quantidade de artigos publicados dentro da área são: "Forensic Science International", "Journal of Forensic Odonto-Stomatology", "Journal of Forensic Science" e "Journal of Forensic Dental Science", todas voltadas para as Ciências Forenses e classificadas como A2, B2, B2 e B3 respectivamente. Entretanto, a revista que se destaca com classificação Qualis A1 é a Journal of Dentistry, apesar de seu baixo número de publicações em Odontologia Legal, totalizando apenas 10 artigos dentre seus 68 volumes com temas em torno de todas as áreas da Odontologia. 


\section{DISCUSSÃO}

A comunicação científica é a forma de estabelecer o diálogo com os pesquisadores, ou seja, a conexão entre os pares por meio de canais de comunicação heterogêneos. As revistas científicas constituem o canal formal da ciência, consideradas como a expressão máxima da autoria das descobertas científicas. É um processo que se inicia com a pesquisa e termina com as descobertas incorporadas ao conhecimento científico, sendo a informação parte inseparável da pesquisa ${ }^{13}$.

A divulgação científica, entretanto, visa à comunicação para o público diversificado, fora da comunidade científica ${ }^{1}$. Vale observar que o número de indivíduos interessados nos assuntos de ciência vem crescendo atualmente, ajudando a consolidar nova configuração nas formas de apropriação do conhecimento. Isto pode ser devido à facilidade atual do acesso à informação, à quantidade de documentos gerados e disponibilizados em rede, ao crescente aumento do número de revistas científicas on-line observado nos últimos anos, e às iniciativas de acesso livre (Open Access) ${ }^{14}$.

O mundo acadêmico e o conhecimento científico legitimado pelo sistema formal de comunicação da ciência, cuja expressão máxima é o periódico científico, passam a conviver com uma forma de comunicação e informação diferente que extrapola o convencional, rompendo fronteiras, ampliando a audiência e fazendo uma grande interseção com públicos não especializados ${ }^{1,15}$.
$\mathrm{Na}$ área da Odontologia, o número de publicações do Medline aumentou exponencialmente nos últimos anos. Observamos que entre os anos 2001 e 2003 o número de publicações foi de 758 e já em todo o século passado, o número total foi de 423. Sendo assim, é inquestionável a importância da divulgação do conhecimento produzido para a sustentação do trabalho científico ${ }^{16}$.

Entretanto, podemos constatar que as categorias temáticas das revistas científicas ligadas à Odontologia, possuem uma visão tecnicista, ou seja, a técnica é supervalorizada, enquanto sua inserção em um contexto social, ético e político são ignorados. Exemplos dessa conjuntura são os artigos publicados na Revista Brasileira de Odontologia, os quais, entre os anos de 1990 e 2004, apresentaram 18,13\% de temas relacionados à Endodontia e 1,49\% sobre Ética e Odontologia Legal, o que pode ser explicado, segundo Péret \& Lima, pela visão mercantilista e interesse privado, apesar da importância do conhecimento sobre legislações e normativas visto a elaboração de documentos odontológicos, como encaminhamentos e atestados ${ }^{6,17}$.

Durante a presente pesquisa, foram encontradas 191 revistas, das quais 75 são nacionais e 116 Internacionais. Todas elas apresentaram temas relacionados à Odontologia Legal em seus objetivos e escopo ou em suas publicações. Entretanto, $33,16 \%$ destas revistas que, teoricamente, visam aceitar artigos nessa área de concentração, no momento da realização dessa pesquisa, não possuem trabalhos publicados na referida especialidade. 
Adicionalmente, as revistas brasileiras com maior número de publicações relacionadas à Odontologia Legal possuem baixas classificações Qualis mostrando, assim, a escassa publicação de artigos científicos em revistas com alto fator de impacto. Já as revistas internacionais apresentam melhor classificação Qualis, entretanto, é possível observar que o número de artigos na área representa uma pequena parcela do total de artigos publicados.

Paralelamente, o "Journal of Forensic Dental Science" e o "Journal of Forensic Odonto-Stomatology" constituem as revistas internacionais com maior quantidade de publicações dentro da área, certamente por serem as mais específicas e com melhor classificação, com Qualis B3 e B2, respectivamente.

Ainda que a Odontologia brasileira apresente altos índices de crescimento em sua produção bibliográfica, há grandes diferenças entre os perfis dos periódicos brasileiros e do exterior, visto a falta de profissionalização do processo editorial pela dificultosa formação de uma equipe especializada, além da restrita adoção de recursos editoriais e uso de softwares para detecção de plágios. Essas e outras características dos periódicos nacionais, como a não indexação em bases de dados de maior prestígio, os distancia dos pares internacionais, diminuindo a visibilidade diante destes e dificultando o vínculo com artigos de alto potencial, mesmo de autores brasileiros ${ }^{18}$.

Além desses fatores, há ainda as chamadas "revistas predatórias", as quais enviam convites para a submissão de artigos e os publicam mediante pagamento, geralmente com um valor mais baixo que as revistas de acesso aberto, mesmo sem a revisão editorial e consequentemente, com baixo controle de qualidade ${ }^{19}$.

Sendo assim, com a diminuição do fator de impacto em bases de dados reconhecidas, da periodicidade, competitividade e internacionalidade, a classificação Qualis é reduzida, justificando essa diferença entre as revistas com maior enfoque em Odontologia Legal ${ }^{4}$.

Por conseguinte, os autores de artigos que abrangem a bioética e a legislação brasileira na Odontologia possuem dificuldades em publicar seus trabalhos em periódicos com alto fator de impacto e competitividade, uma vez que as revistas nacionais sofrem de falta de visibilidade internacional e, revistas publicadas no exterior, muitas vezes não possuem interesse em leis, conjuntos de normas e códigos nacionais que regem a ética e as penas aplicadas no país, visto a distinção frente às legislações particulares de outras nações, e consequentemente, as penalidades podem ser mais ou menos severas $^{18,20,21}$.

Outros fatores a serem considerados são os custos envolvidos para o desenvolvimento da pesquisa forense e as dificuldades nos processos de identificação após ações destrutivas e degenerativas nos corpos das vítimas, bem como a ausência de registos ante mortem. A identificação humana é baseada na comparação de registros ante mortem de uma pessoa conhecida e dados post mortem de um corpo desconhecido. No entanto, as possíveis identificações e estudos tornam- 
se obscuros quando não há registros para comparação ${ }^{22,23}$.

O sistema de avaliação dos

Programas de Pós-Graduação (Mestrado e

Doutorado) tende a adotar o princípio do "ranking", baseado em coeficientes e parâmetros numéricos indicativos de produtividade científica, para orientar uma série de ações políticas dentro das instituições. A proporção de artigos publicados em cada classificação do sistema Qualis favorecerá ou prejudicará essa avaliação, devido à visibilidade da produção científica do país. Assim, é possível concluir que as dificuldades envolvidas para o desenvolvimento de pesquisas forenses e limitações para publicação em periódicos nacionais $\mathrm{e}$ internacionais com alto fator de impacto, reduzem a avaliação dos Programas de Pós-Graduação voltados para a área de Odontologia Legal ${ }^{24,25}$.

\section{CONCLUSÃO}

Foi possível mapear as revistas cientificas com publicações na área da Odontologia Legal, expondo o baixo valor de impacto dos periódicos nacionais em comparação aos internacionais. Estes, apesar de apresentarem um fator de impacto maior, o número de artigos voltados a área é menor, provavelmente devido à distinção das legislações e protocolos particulares de cada nação. Visto isso, fica clara a necessidade de um planejamento estratégico das revistas científicas, considerando a qualidade da informação científica $e$ as diferentes áreas de concentração com as suas consequentes dificuldades e assim, planejar estratégias operacionais e políticas, envolvendo profissionalização editorial e maior participação internacional.

\section{ABSTRACT}

Scientific papers look for the information transference and construction of high quality knowledge. Forensic Dentistry, although the wide field of professional activity, involves several themes related to Law that often makes it difficult to publish in international scientific journals. This study aimed to map the national and international scientific journals with publications in Forensic Dentistry, as well as to expose the difficulties to publish papers in high impact factor journals. It was accessed the Sucupira Platform (Brazil) and selected Dentistry as an evaluation area to get the classification file. All journals that presented issues related to Forensic Dentistry were recorded in two tables with the number of papers published related to the area in each of them. 191 journals were found, 75 national and 116 international. The national publications showed few productions in high impact factor journals, while the international ones presented a larger quantity, but still a minority within the total production. With this study, it was possible to compare the Qualis classification between national and international journals, highlighting the distance between such classifications and exposing the difficulty of publication in journals that present high competitiveness and internationality.

\section{KEYWORDS}

Forensic dentistry; Impact factor; Legislation; Periodicals; Research.

\section{REFERÊNCIAS}

1. Silveira MSM, Oddone NE. Livre acesso à literatura científica: realidade ou sonho de cientistas e bibliotecários? Anais do V Cinform. 2004.

2. Marcuschi LA. Revistas Brasileiras em Letras e Linguística. DELTA. 2001; 17(especial): 83-120.
3. Stumpf IRC. Passado e Futuro das Revistas Científicas. Ciência da Informação. 1996; 25(3).

4. Plataforma Sucupira: Qualis-Periódicos. Disponível em: https://sucupira.capes.gov.br/sucupira. Acesso em: 07 de fevereiro de 2018. 
5. Andrade RO. Para publicar com critério. Pesquisa FAPESP. 2016; 244: 96-98.

6. Amorim KPC, Alves MSCF, Germano RM, Costa ICC. A construção do saber em Odontologia: a produção científica de três periódicos brasileiros de 1990 a 2004. Interface - Comunic, Saúde, Educação. 2007; 11(21): 9-23.

7. Ribas-e-Silva V, Terada ASSD, Silva RHA. A importância do conhecimento especializado do cirurgião-dentista nas equipes de perícia oficial do Brasil. Rev Bras de Odontol Leg RBOL. 2015; 2(1): 68-90.

http://dx.doi.org/10.21117/rbol.v2i1.22.

8. Carvalho MSOA. A importância da Documentação Odontológica. Revista Odonto News. 2006.

9. Brasil. Conselho Federal de Odontologia. Resolução CFO-63/2005: Consolidação das Normas para Procedimentos nos Conselhos de Odontologia. Disponível em: http://cfo.org.br/wpcontent/uploads/2009/10/consolidacao.pdf. Acesso em: 07 de fevereiro de 2018.

10. Almeida CA, Paranhos LR, Silva RHA. A importância da Odontologia na identificação post-mortem. Odontologia e Sociedade. 2010; 12(2): 07-13.

11. US National Library of Medicine (NLM): NLM Catalog. Disponível em: https://www.ncbi.nlm.nih.gov/nlmcatalog/. Acesso em: 7 de fevereiro de 2018.

12. US National Library of Medicine (NLM): PubMed. Disponível em: https://www.ncbi.nlm.nih.gov/pubmed/. Acesso em: 07 de fevereiro de 2018.

13. Valerio PM, Pinheiro LVR. Da comunicação científica à divulgação. TransInformação. 2008; 20(2): 159-169.

14. Silva HC. O que é divulgação científica? Ciência \& Ensino. 2006; 1(1): 53-59.

15. Vanz SAS, Stumpf IRC. Colaboração científica: revisão teórico-conceitual. Perspectivas em Ciência da Informação. 2010; 15(2): 42-55.

16. Cury J. The evolution of dental research in Brazil. Brazilian Dental Research. 2004; 18(2): 1.

17. Peret ACA, Lima MLR. A pesquisa e a formação do professor de Odontologia nas políticas internacionais e na educação. Rev ABENO. 2003; 3(1): 65-9.

18. Shinkai R. O cenário atual dos periódicos brasileiros de odontologia. RFO Passo Fundo. 2011; 16(3): 242-3.

19. Rodrigues E. O acesso aberto (na UMinho e no mundo): onde estamos e por onde vamos? RECIIS. 2014; 8(2): 180-194.
20. Pires LAG, Cerveira J. A Bioética na Odontologia. Stomatos. 2003; 9(17): 7-12.

21. Brasil. Ministério das Relações Exteriores Portal Consular. http://www.portalconsular.itamaraty.gov.br/l egislacao-do-brasil. Acesso em: 07 de fevereiro de 2018.

22. Araújo LG, Biancalana RC, Terada ASSD, Paranhos LR, Machado CEP, Silva RHA. Human identification of victims of mass disasters: the importance and role of Forensic Dentistry. RFO. 2013;18(2):224-9.

23. Carneiro APC, Andrade LM, Fraga FJO, Duarte Ml. Aplicação dos métodos de identificação humana post mortem no IML Estácio de Lima no período de janeiro de 2011 a dezembro de 2015. Perspectivas. 2017;4. Disponível em: http://perspectivas.med.br/2017/10/aplicac ao-dos-metodos-de-identificacao-humanapost-mortem-no-iml-estacio-de-lima-noperiodo-de-janeiro-de-2011-a-dezembrode-2015-2/.

24. Barata RCB. Ten things you should know about the Qualis. RBPG. 2016 13(30): 1340.

25. Santana GA, Sobral NV, Ferreira MHW, Silva FM. Indicadores Científicos: Uma Análise da Produção do Programa de PósGraduação em Sociologia (PPGS) da UFPE a partir dos currículos da Plataforma Lattes (PL). Anais da XIV EREBD UFMA. 2011. 